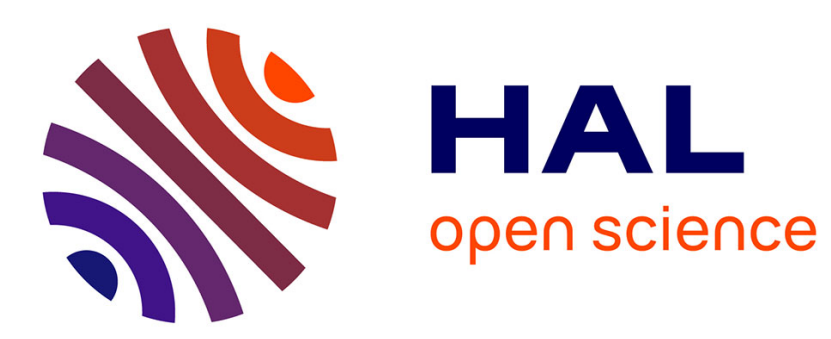

\title{
Analytical seven-wave model for wave propagation in a degenerate dual-pump fiber phase sensitive amplifier
}

Debanuj Chatterjee, Yousra Bouasria, Fabienne Goldfarb, Fabien Bretenaker

\section{To cite this version:}

Debanuj Chatterjee, Yousra Bouasria, Fabienne Goldfarb, Fabien Bretenaker. Analytical seven-wave model for wave propagation in a degenerate dual-pump fiber phase sensitive amplifier. Journal of the Optical Society of America B, 2021, 10.1364/JOSAB.417542 . hal-03162876

\section{HAL Id: hal-03162876 https://hal.science/hal-03162876}

Submitted on 11 Mar 2021

HAL is a multi-disciplinary open access archive for the deposit and dissemination of scientific research documents, whether they are published or not. The documents may come from teaching and research institutions in France or abroad, or from public or private research centers.
L'archive ouverte pluridisciplinaire HAL, est destinée au dépôt et à la diffusion de documents scientifiques de niveau recherche, publiés ou non, émanant des établissements d'enseignement et de recherche français ou étrangers, des laboratoires publics ou privés. 


\title{
An analytical 7-wave model for wave propagation in a degenerate dual-pump fiber phase sensitive amplifier
}

\author{
Debanuj Chatterjee ${ }^{1}$, Yousra Bouasria ${ }^{2}$, Fabienne Goldfarb ${ }^{1}$, and Fabien Bretenaker ${ }^{1,3} 3^{* *}$ \\ ${ }^{1}$ Université Paris-Saclay, CNRS, ENS Paris-Saclay, CentraleSupélec, LuMIn, Gif-sur-Yvette, France \\ ${ }^{2}$ Equipe Sciences de la Matière et du Rayonnement (ESMaR), Faculté des Sciences, Université Mohammed V, Rabat, Morroco \\ ${ }^{3}$ Light and Matter Physics Group, Raman Research Institute, Bangalore 560080, India \\ *Corresponding author: fabien.bretenaker@universite-paris-saclay.fr
}

Compiled March 8, 2021

\begin{abstract}
We develop an analytical model to describe propagation of seven continuous (CW) waves (two strong pumps, a degenerate signal and idler, two high-order idlers (HOIs) and two high-order pumps (HOPs)) through a nonlinear fiber. The model is developed considering the pumps to be much stronger than the other waves. The 7-wave system is analyzed in terms of interactions between its 4-wave subsystems : (a) pumps and degenerate signal and idler, (b) pumps and HOIs, and (c) pumps and HOPs. First we analyze the three 4-wave subsystems, and then we move to the 7-wave system and compare the two analytical models. The analytical 7-wave model reveals that a strong coupling (mediated through four wave mixing processes), between the subsystem with the signal and the subsystem with the HOIs leads to an important role of the HOIs in influencing the signal gain of a degenerate dual-pump fiber phase sensitive amplifier (PSA). We find that the maximum PSA gain of the signal for such an amplifier can be significantly enhanced by launching the HOIs at the fiber input along with the signal. We compare the analytical results with that of a numerical 7-wave model and for the anomalous dispersion regime we find a good agreement between the two when the system nonlinearity is weak, i.e. total nonlinear phase less than 0.6 rad. (C) 2021 Optical Society of America
\end{abstract}

http://dx.doi.org/10.1364/ao.XX.XXXXXX

\section{INTRODUCTION}

Fiber-optic parametric amplifiers (FOPA) may play a vital role in future communication systems. In particular, fiber phase sensitive amplifiers (PSA) offer promising functionalities such as high gain and ultra-low noise properties [1-5], which make them a potential candidate for a variety of applications such as microwave photonics [6-8], 5G technology [9], long-haul optical communication systems $[10,11]$, etc. Such optical amplifiers rely on the parametric amplification of a signal by a strong pump mediated by the nonlinear optical interaction of the pump with the signal, while propagating through a highly nonlinear fiber (HNLF) [12].

A FOPA can be operated in different configurations. The most common configurations are i) single pump with non-degenerate signal and idler, and ii) two pumps with a degenerate signal and idler. From an application point of view, the latter one is often preferred $[5,13,14]$. This is because, on the one hand, the degenerate signal and idler configuration eliminates the requirement of an extra idler wave in the single pump configuration that needs to be phase-locked with the signal [15-17]. On the other hand, the dual-pump configuration provides a flatter gain spectrum compared to the single pump one. However, one dis- advantage of the dual-pump configuration is that, due to the presence of two strong pumps, when the wavelength separation between the pumps becomes small, cascaded four-wave mixing (FWM) processes lead to the generation of high-order sidebands that often deplete the available gain of the FOPA. Therefore it is imperative to understand the physics of the system that gives rise to the parasitic waves in order to improve the amplifier performance.

Initial theoretical investigations of this system started with the investigation of the coupled differential equations that govern the power evolution of four waves (signal, idler and two strong pumps) $[18,19]$. Subsequently, Chen and Snyder solved the power evolution of the 4-wave system incorporating pump depletion [20]. Inclusion of the pump depletion effects complicates the analysis significantly, and the solution is expressed in terms of elliptic or hyperbolic Jacobian functions [19, 21]. Further, the nonlinear evolution of such systems were also investigated [22-24]. Numerical investigations of the 6-wave (two pumps, signal, idler and two high-order idlers) model were performed by Marhic et al. [25] and Vedadi et al. [26]. The 6-wave model was solved by Marhic et al. neglecting fiber dispersion $[27,28]$. Tanemura et al. studied the 6-wave system in terms of 
its eigenmodes [29]. Pakarzadeh and Zakery investigated the 6-wave model numerically for optimization of the shape of the gain spectra [30,31]. Ignoring nonlinear effects of the fiber, a dispersive 6-wave system was studied by Silva et al. [32]. In the context of PSA noise figure calculations, McKinstrie et al. analyzed the 6-wave model, however providing an analytical solution under certain special cases only [33]. Such 6-wave systems were experimentally investigated by Albuquerque et al. [34].

Recently, the degenerate signal-idler configuration was $n u$ merically investigated by Xie et al. in the framework of the 7-wave model [35]. Pushing the limit further, Qian et al. numerically solved the coupled equations for a dual pump PSA for as much as 27 waves [36]. Note that with the increase of the number $n$ of involved waves, the total number of FWM terms in the coupled differential equations governing the wave evolution increases as $n^{3}$ [37], making the system more and more complicated. Inoue developed a semi-analytical model to solve for the two pumps and high-order pumps (HOPs) numerically while incorporating their effects on the signal and high-order idlers (HOIs) analytically [38]. However, Inoue's approach does not incorporate all the important FWM processes that influence the signal propagation through a FOPA [39].

It thus appears that although the multiwave models have been studied extensively both analytically and numerically, no complete analytical solution is available beyond the 4-wave model. Following Xie et al., who predicted the possibility of a large signal PSA gain using the 7-wave model numerically, in the present paper, we derive an analytical 7-wave model to investigate the physics of a PSA system in dual-pump degenerate signal and idler configuration.

This paper is organized as follows. In Section 2 we introduce the general formalism for multiple $\mathrm{CW}$ wave propagation in a nonlinear fiber and define some important physical quantities to be used later. Then in Section 3, we use the general formalism to describe propagation of just four waves, i.e. two strong pumps, a signal and an idler. The analytical 4-wave model is introduced at this point, as later these results will be used in describing an analytical 7-wave model. Then in Section 4, similar to Section 3 , we extend the formalism to the case of seven waves, i.e. two strong pumps, degenerate signal and idler, two HOIs and two HOPs. We analyze the 7-wave system by deconstructing it into its constituent 4-wave subsystems. Finally in Section 5 we outline the limitations of our model, project some perspectives for future work, and summarize the important results obtained.

\section{MODEL PROPERTIES}

In our model we consider a system with $2 k+1$ equally spaced $C W$ waves with two strong pumps and degenerate signal and idler. In Fig. 1 we show such a configuration, where any wave is indexed with respect to a dimensionless frequency parameter $q$, given as :

$$
q=\frac{2\left(\omega-\omega_{0}\right)}{\Delta \omega_{\mathrm{PP}}}
$$

where $\omega$ is the frequency of the considered wave, $\omega_{0}$ is the frequency of the signal, and $\Delta \omega_{\mathrm{PP}}$ is the frequency separation of the two pumps.

We write down the total scalar real electric field $E(z, t)$ of the $2 k+1 \mathrm{CW}$ waves as :

$$
E(z, t)=\sum_{j=-k}^{k} A_{j}(z) e^{i\left(\beta\left(\omega_{j}\right) z-\omega_{j} t\right)}+\text { c.c., }
$$

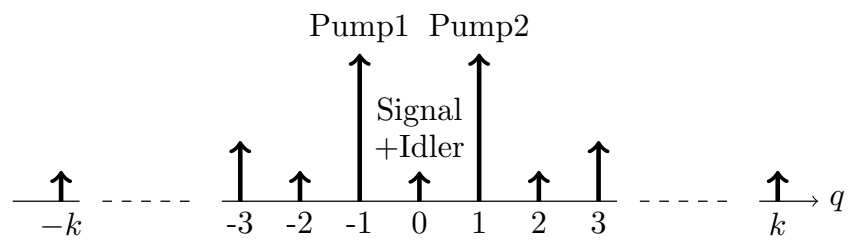

Fig. 1. Configuration of a dual-pump FOPA with degenerate signal and idler, consisting of $2 k+1 \mathrm{cw}$ waves.

where $A_{j}$ is the complex amplitude, $\beta\left(\omega_{j}\right)$ is the propagation constant and $\omega_{j}$ is the frequency of the wave with index $j$. We have considered that all the waves are co-polarized along the length of the fiber and neglected the variation of the field in the transverse $(x-y)$ direction.

The coupled differential equations for the evolution of $2 k+1$ slowly varying amplitudes, $A_{j}$ 's with $j=-k$ to $k$, along the fiber, neglecting fiber attenuation, are given as [28] :

$$
\begin{gathered}
\frac{d A_{j}}{d z=}+i \gamma\left[\left|A_{j}\right|^{2} A_{j}+2 \sum_{l \neq j=-k}^{k}\left|A_{l}\right|^{2} A_{j}\right. \\
\left.+\sum_{\substack{m, n, p=-k, \omega_{m}+\omega_{n}-\omega_{p}=\omega_{j}}}^{k} A_{m} A_{n} A_{p}^{*} e^{i \Delta \beta_{m n p j} z}\right],
\end{gathered}
$$

where $\Delta \beta_{\text {mnpj }}=\beta\left(\omega_{m}\right)+\beta\left(\omega_{n}\right)-\beta\left(\omega_{p}\right)-\beta\left(\omega_{j}\right)$ is the linear phase mismatch coefficient, and $\gamma$ is the nonlinear coefficient of the fiber. The three terms on the right-hand side of Eq. (3) respectively correspond to: i) the interaction of one wave, $A_{j}$ with itself, which is called self-phase modulation (SPM); ii) the interaction between two waves, $A_{l}$ and $A_{j}$, where $l \neq j$, which is called cross-phase modulation (XPM); iii) the interaction between four waves, $A_{m}, A_{n}, A_{p}$ and $A_{j}$, where the energy conservation constraint leads to $\omega_{m}+\omega_{n}=\omega_{p}+\omega_{j}$, which is called four wave mixing (FWM). The SPM and XPM interactions between the waves only account for a phase shift of the waves, while the FWM interactions lead to an exchange of energy between the different waves [12, 28]. The FWM interaction is the mechanism that is central to the design of high-gain optical parametric amplifiers as it leads to energy exchanges between the four waves it involves [5].

\section{A. Linear Phase Mismatch Coefficient}

As mentionned above, the linear phase mismatch coefficient $\Delta \beta_{m n p j}$ between four interacting waves at frequencies $\omega_{m}, \omega_{n}$, $\omega_{p}$ and $\omega_{j}$ is given by :

$$
\Delta \beta_{m n p j}=\beta\left(\omega_{m}\right)+\beta\left(\omega_{n}\right)-\beta\left(\omega_{p}\right)-\beta\left(\omega_{j}\right) .
$$

We can rewrite $\beta(\omega)$ by performing a Taylor series expansion around a central frequency $\omega_{\mathcal{c}}$ and truncating the series after second order terms as :

$$
\beta(\omega) \approx \beta\left(\omega_{c}\right)+\left(\frac{d \beta}{d \omega}\right)_{\omega_{c}}\left(\omega-\omega_{c}\right)+\left(\frac{d^{2} \beta}{d \omega^{2}}\right)_{\omega_{c}} \frac{\left(\omega-\omega_{c}\right)^{2}}{2} .
$$

Using Eq. (5) in Eq. (4), and using the energy conservation criterion, i.e. $\omega_{m}+\omega_{n}-\omega_{p}-\omega_{j}=0$, we calculate $\Delta \beta_{m n p j}$ as :

$$
\Delta \beta_{m n k j}=\frac{\beta^{(2)}\left(\omega_{c}\right)}{2}\left(\omega_{m}^{2}+\omega_{n}^{2}-\omega_{k}^{2}-\omega_{j}^{2}\right),
$$


where $\beta^{(2)}\left(\omega_{c}\right)=\left(\frac{d^{2} \beta}{d \omega^{2}}\right)_{\omega_{c}}$. Without loss of generality, we choose $\omega_{c}=\omega_{0}$. Thus, in terms of the frequency parameters $q_{m}, q_{n}, q_{p}$ and $q_{j}$ of the waves at frequencies $\omega_{m}, \omega_{n}, \omega_{p}$ and $\omega_{j}$ respectively (see Eq. (1)), $\Delta \beta_{m n p j}$ can be expressed as :

$$
\Delta \beta_{m n p j}=C\left(q_{m}^{2}+q_{n}^{2}-q_{p}^{2}-q_{j}^{2}\right),
$$

where $C$ is given by :

$$
C=\frac{\beta^{(2)}\left(\omega_{0}\right) \Delta \omega_{\mathrm{PP}}^{2}}{8} .
$$

For a given fiber, $\beta^{(2)}\left(\omega_{0}\right)$ can be obtained from the following relation [12] :

$$
\beta^{(2)}\left(\lambda_{0}\right)=-\frac{\lambda_{0}^{2} D^{\prime}\left(\lambda_{0}-\lambda_{\mathrm{ZDW}}\right)}{2 \pi c},
$$

where $\lambda_{0}=2 \pi c / \omega_{0}$ is the signal wavelength and $c$ is the speed of light in vacuum. $\lambda_{\mathrm{ZDW}}$ and $D^{\prime}$ are the zero-dispersion wavelength and dispersion slope of the fiber, respectively.

\section{B. Total Effective Phase Mismatch}

It is evident from the above discussion, in particular from Eq. (3), that the $z$-evolution of the system depends on the different FWM processes that redistribute energy among the different waves in the system. Therefore it is important to quantify the efficiency of the different FWM processes in terms of the system parameters. To this aim, first we define the effective phase mismatch coefficient $\kappa_{m n p j}$ as [35] :

$$
\kappa_{m n p j}=\Delta \beta_{m n p j}-\gamma P_{m n p j},
$$

where $\gamma P_{\text {mnpj }}$ is the nonlinear phase mismatch coefficient and $P_{m n p j}$ is given by:

$$
P_{m n p j}=P_{m}+P_{n}-P_{p}-P_{j} .
$$

$P_{i}$ is the power of the wave with frequency parameter $i$, evaluated at the end of the fiber. The total effective phase mismatch $\kappa_{\text {tot,mnpj }}$ of the FWM process occurring between the waves at frequencies $\omega_{m}, \omega_{n}, \omega_{p}$ and $\omega_{j}$ (denoted as $p_{m n p j}$ ) is the product of the effective phase mismatch coefficient $\kappa_{m n p j}$ and the fiber length $L$ :

$$
\kappa_{t o t, m n p j}=\kappa_{m n p j} L
$$

$\kappa_{\text {tot,mnpj }}$ provides an estimate of the efficiency of the process $p_{m n p j}$ along the fiber [18]. An efficient FWM is possible only when $\left|\kappa_{\text {tot,mnpj }}\right|$ is close to 0 . A value of $\left|\kappa_{t o t, m n p j}\right|$ near or larger than $\pi$ implies an inefficient occurrence of the process $p_{m n p j}$ [12].

\section{Strong Pump Approximation}

In our model, the pumps are considered to be much more powerful than the other waves along the entire length of the fiber. We also consider the input powers of the two pumps to be the same, i.e. $\left|A_{1}(0)\right|^{2}=\left|A_{-1}(0)\right|^{2}$, and to remain undepleted along the fiber. These undepleted pump powers are given by $\left|A_{ \pm 1}(z)\right|^{2}=P$. Notice that here we consider a re-scaled system of units where the modulus square of the complex field amplitude of a given wave gives its power in Watts. We stick to this convention throughout this paper.

\section{Pump Equations}

Considering the strong pump approximation, the following pump evolution equations are obtained from Eq. (3) :

$$
\frac{d A_{ \pm 1}}{d z}=i \gamma 3 P A_{ \pm 1}
$$

where the terms that are product of less than three pump amplitudes $\left(A_{ \pm 1}\right.$ or $\left.A_{ \pm 1}{ }^{*}\right)$ have been neglected. Therefore the evolution of the pump waves is given as:

$$
A_{ \pm 1}(z)=\sqrt{P} e^{i \gamma 3 P z}
$$

where we have taken the initial phases of the pumps to be 0 . We note here that this specific input phase allocation of the pumps does not influence our analysis. This is because, from the literature, we know that the PSA mechanism depends on the relative input phases of the two pumps and the signal [12]. The choice of the phases of the two pumps is thus completely arbitrary.

\section{E. System Parameters}

Unless stated otherwise, in all the following discussion, we use a set of system parameters similar to those used in Ref. [35]. The main four-wave mixing process, involving the degenerate signal and idler and the two pumps, denoted $p_{1-100}$, would be perfectly phase matched when the signal wavelength lies in the normal dispersion region of the fiber. However, we choose here to focus instead on the anomalous dispersion regime. Indeed, following Ref. [35], we expect this regime to lead to a strong enhancement of the gain with respect to the 3-wave approach, showing that many other FWM processes are involved beyond the basic $p_{1-100}$ process. By focusing on this anomalous dispersion region, we thus intend to compare our analytical approach with the numerical approach of Ref. [35], in order to assess the validity of the present theory. However, the present theory could be equally well applied to the normal dispersion case.

We thus consider a standard highly nonlinear fiber (HNLF) with $\gamma=11.3 \mathrm{~W}^{-1} \cdot \mathrm{km}^{-1}, \lambda_{\mathrm{ZDW}}=1547.5 \mathrm{~nm}, D^{\prime}=$ $0.017 \mathrm{ps} . \mathrm{nm}^{-2} \cdot \mathrm{km}^{-1}$ and fiber length $L=500 \mathrm{~m}$. We use a pump power $P=20 \mathrm{dBm}$ and a central wavelength $\omega_{c}=1557.5$ nm.

\section{ANALYTICAL 4-WAVE MODEL}

Although our final goal is to develop an analytical 7-wave model (as shown in Fig. 1 with $|k|=3$ ), in this section, we take a detour, and first describe an analytical 4-wave model with two pumps, a signal, and an idler (see Fig. 2). The motivation for analyzing such a 4-wave model is to understand the evolution of a simpler 4-wave system compared to the more complex 7-wave system. Thus, later in the 7-wave framework, we will be able to describe the system dynamics with respect to its 4-wave simpler subsystems, as we will discuss in Section 4.

The analytical solution for the 4 -wave situation of Fig. 2 is easy to find in the literature $[12,28]$, except in the situation where the frequency separation between the signal and the pump is equal to the frequency separation between the two pumps. Indeed, in this situation, corresponding to $|s|=3$ in Fig. 2, extra FWM processes have to be taken into account, compared to the ordinary situation $|s| \neq 3$. In the present section, we give a general derivation that can apply to both cases. This will be needed when we deal with the 7-wave model in Section 4, which will be 


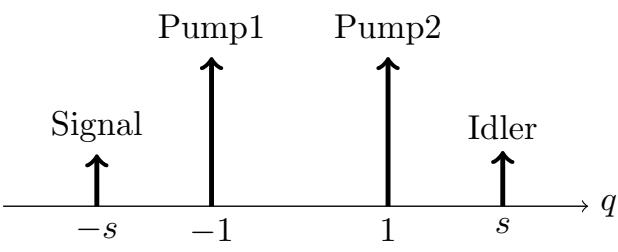

Fig. 2. Configuration of a 4-wave model with arbitrary allocation of signal frequency. The signal frequency parameter is $q=-s$.

shown to break into several 4-wave systems, some with $|s|=3$, and some with $|s| \neq 3$.

In the considered analytical 4-wave model, note that the signal $(q=-s)$ and the idler $(q=s)$ are non-degenerate in general, except when $s=0$, which then corresponds to a 3-wave model (two pumps and a degenerate signal and idler as in Fig. 1 with $|k|=1)$.

To determine the evolution equations for $A_{S}$ and $A_{-s}$, first we invoke the strong pump approximation, i.e. neglect all terms that are product of less than two pump amplitudes $\left(A_{ \pm 1}\right.$ or $\left.A_{ \pm 1}{ }^{*}\right)$ in Eq. (3). Note that Eq. (3) is still valid for this configuration however now, the index $j$ takes the values $j=-s,-1,1$, s. Next we inject the pump solutions Eq. (14) into Eq. (3). We calculate the relevant linear phase mismatch coefficients $\left(\Delta \beta_{m n p j}\right.$ 's) using Eq. (7). Finally we express the equations of evolution of the amplitudes of the signal $A_{-s}$ and idler $A_{s}$ in a matrix form as :

$$
\begin{aligned}
\frac{d}{d z}\left[\begin{array}{c}
A_{s} \\
A_{-s}{ }^{*}
\end{array}\right] & =i \gamma P\left[\begin{array}{cc}
4 & 2 e^{i\left(6 \gamma P+2 C\left(1-s^{2}\right)\right) z} \\
-2 e^{-i\left(6 \gamma P+2 C\left(1-s^{2}\right)\right) z} & -4
\end{array}\right]\left[\begin{array}{c}
A_{s} \\
A_{-s}{ }^{*}
\end{array}\right] \\
& +\sigma(s)\left[\begin{array}{c}
i \gamma P^{\frac{3}{2}} e^{i\left(3 \gamma P+C\left(1-s^{2}\right)\right) z} \\
-i \gamma P^{\frac{3}{2}} e^{-i\left(3 \gamma P+C\left(1-s^{2}\right)\right) z}
\end{array}\right],
\end{aligned}
$$

where $\sigma(s)$ is given by :

$$
\sigma(s)=\left\{\begin{array}{l}
0 \text { when }|s| \neq 3 \\
1 \text { when }|s|=3
\end{array}\right.
$$

Equation (15) leads to a few important observations. First, when $|s|=3$, the system of equations becomes inhomogeneous, whereas remaining homogeneous for all other values of $s$. This is because, when $|s|=3$, the configuration attains an extra degree of symmetry that leads to an extra FWM process involving three pump amplitudes. This is illustrated in Fig. 3. The FWM process $p_{1-1 s-s}$ is present both for $|s| \neq 3$ and $|s|=3$ (see red curved arrows in Figs. 3 (a) and (b)). However, the processes $p_{-1-11-s}$ and $p_{11-1 s}$ are only possible when $|s|=3$ (see blue and green curved arrows in Fig. 3 (c)). The reason behind this is that this extra FWM process, where two pump photons get converted to a signal (or idler) and a pump photon, is possible only when energy conservation is satisfied, i.e. $\hbar \omega_{-1}-\hbar \omega_{-s}=\hbar \omega_{1}-\hbar \omega_{-1}$ or $\hbar \omega_{1}-\hbar \omega_{s}=\hbar \omega_{-1}-\hbar \omega_{1}$.

Second, under the strong pump approximation, Eq. (3), which is nonlinear in general, leads to a linear system of ODEs, i.e. Eq. (15). Also, the homogeneous part of Eq. (15) has the same form as is used to describe the dynamics of an oscillating pendulum of time-varying length, or that of a child's swing pumped by the squatting and rising motion of the child [40]. Such parametric oscillations have been extensively studied in the context of nonlinear optics, especially in studying the dynamics of optical parametric amplifiers [5].

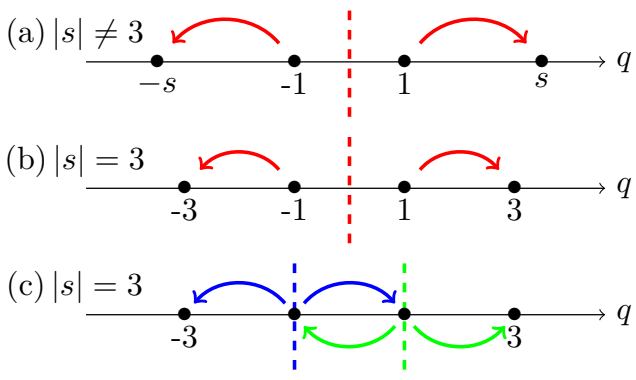

Fig. 3. Illustrations of different symmetry axes (dashed lines) and corresponding FWM processes (curved arrows) for : (a) 4-wave model with $|s| \neq 3$, (b,c) 4-wave model with $|s|=3$. FWM processes corresponding to blue and green arrows are only possible when $|s|=3$.

Note that Eq. (15) is of the form $d \mathbf{A} / d z=\mathbf{M}(z) \mathbf{A}+\mathbf{N}(z)$. Also, in our case, the coefficient matrix $\mathbf{M}(z)$ is periodic in $z$. From Floquet theory we know that such a non-autonomous (i.e. coefficient matrix $\mathbf{M}$ is $z$-dependent) system can be transformed into an autonomous (i.e. coefficient matrix is $z$-independent) one with a suitable transformation of coordinates [41]. We thus perform the following transformation of variables (see the Appendix) :

$$
B_{ \pm s}=e^{-i\left(3 \gamma P+C\left(1-s^{2}\right)\right) z} A_{ \pm s} .
$$

Then Eq. (15) can be rewritten as :

$$
\frac{d}{d z}\left[\begin{array}{c}
B_{s} \\
B_{-s} *
\end{array}\right]=\operatorname{i\gamma P}\left[\begin{array}{cc}
1-F_{s} & 2 \\
-2 & -\left(1-F_{s}\right)
\end{array}\right]\left[\begin{array}{c}
B_{s} \\
B_{-s} *
\end{array}\right]+\sigma(s)\left[\begin{array}{c}
i \gamma P^{\frac{3}{2}} \\
-i \gamma P^{\frac{3}{2}}
\end{array}\right],
$$

where $F_{s}$ is given by :

$$
F_{s}=\frac{C\left(1-s^{2}\right)}{\gamma P} .
$$

The coefficient $F_{S}$ is basically the ratio between the dispersion and nonlinearity of the fiber. The solution of the system relating the input-output modes is given by :

$$
\begin{aligned}
{\left[\begin{array}{c}
B_{s}(z) \\
B_{-s}{ }^{*}(z)
\end{array}\right] } & =\left[\begin{array}{cc}
\cos \left(\gamma P \mu_{s} z\right)-\frac{\eta_{s}}{\mu_{s}} i \sin \left(\gamma P \mu_{s} z\right) & \frac{2}{\mu_{s}} i \sin \left(\gamma P \mu_{s} z\right) \\
-\frac{2}{\mu_{s}} \sin \left(\gamma P \mu_{s} z\right) & \cos \left(\gamma P \mu_{s} z\right)+\frac{\eta_{s}}{\mu_{s}} i \sin \left(\gamma P \mu_{s} z\right)
\end{array}\right]\left[\begin{array}{c}
B_{s}(0) \\
B_{-s}{ }^{*}(0)
\end{array}\right] \\
& +\sigma(s) \sqrt{P}\left[\begin{array}{ll}
\frac{1}{2-\eta_{3}} \cos \left(\gamma P \mu_{3} z\right)+\frac{1}{\eta_{3}-2}+\frac{i}{\mu_{3}} \sin \left(\gamma P \mu_{3} z\right) \\
\frac{1}{2-\eta_{3}} \cos \left(\gamma P \mu_{3} z\right)+\frac{1}{\eta_{3}-2}-\frac{i}{\mu_{3}} \sin \left(\gamma P \mu_{3} z\right)
\end{array}\right]
\end{aligned}
$$

where $\eta_{s}$ and $\mu_{s}$ are given by:

$$
\eta_{s}=-1+F_{s}, \quad \mu_{s}=\left(-3-2 F_{s}+F_{s}^{2}\right)^{\frac{1}{2}} .
$$

In order to discuss the physical meaning of these solutions, we distinguish in the following the cases $|s|=3$ and $|s|=0,2$.

\section{A. Case $1:|s|=3$}

For the case of $|s|=3$, the solution of Eq. (20) has two terms. The first term depends on the initial conditions whereas the second term does not. Under the strong pump approximation, the second term dominates and the solution can be approximated as :

$B_{ \pm 3}(z) \approx \sqrt{P}\left(\frac{1}{2-\eta_{3}} \cos \left(\gamma P \mu_{3} z\right)+\frac{1}{\eta_{3}-2}+\frac{i}{\mu_{3}} \sin \left(\gamma P \mu_{3} z\right)\right)$. 
The above solution implies that, for $|s|=3$, due to the symmetry of the configuration, efficient interaction with the pumps leads to a large phase insensitive gain for the signal and the idler.

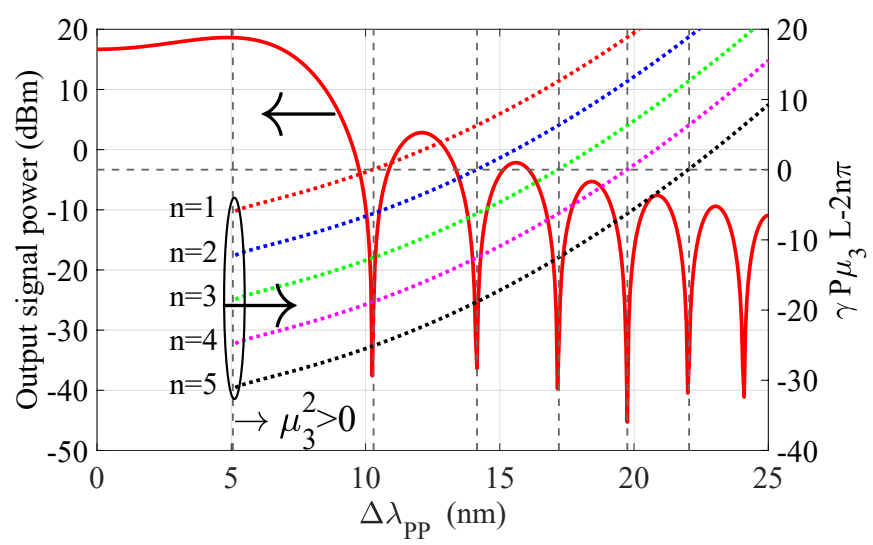

Fig. 4. Red solid line: output signal power for $|s|=3$ versus $\Delta \lambda_{\mathrm{PP}}$. Dotted lines: evolution of $\gamma P \mu_{3} L-2 n \pi$ versus $\Delta \lambda_{\mathrm{PP}}$ when $\mu_{3}^{2}>0$ for $n=1$ (red), 2 (blue), 3 (green), 4 (magenta) and 5 (black). Grey dashed lines are guides for the eye. $P=20$ $\mathrm{dBm}, B_{ \pm 3}(0)=0, \lambda_{c}=1557.5 \mathrm{~nm}, \gamma=11.3 \mathrm{~W}^{-1} \mathrm{~km}^{-1}$, $\lambda_{\mathrm{ZDW}}=1547.5 \mathrm{~nm}, D^{\prime}=0.017 \mathrm{ps} / \mathrm{nm}^{2} / \mathrm{km}$, and $L=500 \mathrm{~m}$.

Since Eq. (22) is a combination of cosines and sines, one can wonder whether one can suppress the signal (and the idler) at the fiber output when $|s|=3$ ? This means that the real and imaginary parts of $B_{ \pm 3}(L)$ should go to 0 . Using Eq. (22) this leads to:

$$
\frac{\sin \left(\gamma P \mu_{3} L\right)}{\mu_{3}}=0, \cos \left(\gamma P \mu_{3} L\right)=1,
$$

where we supposed that $\eta_{3} \neq 2$. Note that these equations can only be satisfied for real values of $\mu_{3}$. Thus the conditions in Eq. (23) lead to :

$$
\gamma P \mu_{3} L=2 n \pi,
$$

where $n \in \mathbb{N}$. Note that here $n=0$ is not allowed since that would mean $\mu_{3}=0$, and $\lim _{\mu_{3} \rightarrow 0} \frac{\sin \left(\gamma P \mu_{3} L\right)}{\mu_{3}}=\gamma P L \neq 0$. The red solid line in Fig. 4 shows the evolution of the output power of the signal (or idler) $\left|B_{ \pm 3}\right|^{2}$ in $\mathrm{dBm}$ (left vertical axis) as a function of the pump-pump wavelength separation $\Delta \lambda_{\mathrm{PP}}$, as obtained from Eq. (22). The input powers of the signal and idler are taken to be 0, i.e. $B_{ \pm 3}(0)=0$. The dashed lines in Fig. 4 represent $\gamma P \mu_{3} L-2 n \pi$ in natural scale (right vertical axis) for $n=1$ (red), 2 (blue), 3 (green), 4 (magenta) and 5 (black) in the region where $\mu_{3}$ is real. The zeroes of all these curves (in dashed lines) correspond to different situations where $B_{ \pm 3}(L)=0$. This phenomenon is also reflected in the dips in the plot of the output power of the signal. Grey dashed lines are used to show the concurrency of the dips of output signal power with the roots of Eq. (24).

The above discussion suggests that by tailoring $\Delta \lambda_{\mathrm{PP}}$ and/or $\gamma P L$, it is possible to achieve a PSA with suppressed output signal power even when $|s|=3$. Such an operation might be useful due to the detrimental nature of the high-order pumps (corresponding to $|s|=3$ ) often observed in a FOPA [36].

\section{B. Case $2:|s|=0,2$}

In the cases where $|s|=0,2$ (or any values of $|s| \neq 3$ ), the signal gain is phase sensitive, as can be seen from the first term in the right-hand side of Eq. (20). Figure 5 shows the maximum (with respect to the input signal phase) PSA signal gain (left vertical axis) as a function of $\Delta \lambda_{\mathrm{PP}}$ for $s=0$ (blue solid line) and $|s|=2$ (red dotted line), evaluated using Eq. (20). In the same figure, we also plot the total effective phase mismatches (right vertical axis) corresponding to the only relevant FWM process $p_{1-1 s-s}$ in the 4-wave model, for $s=0$ (blue dashed line) and $|s|=2$ (red dashed line).

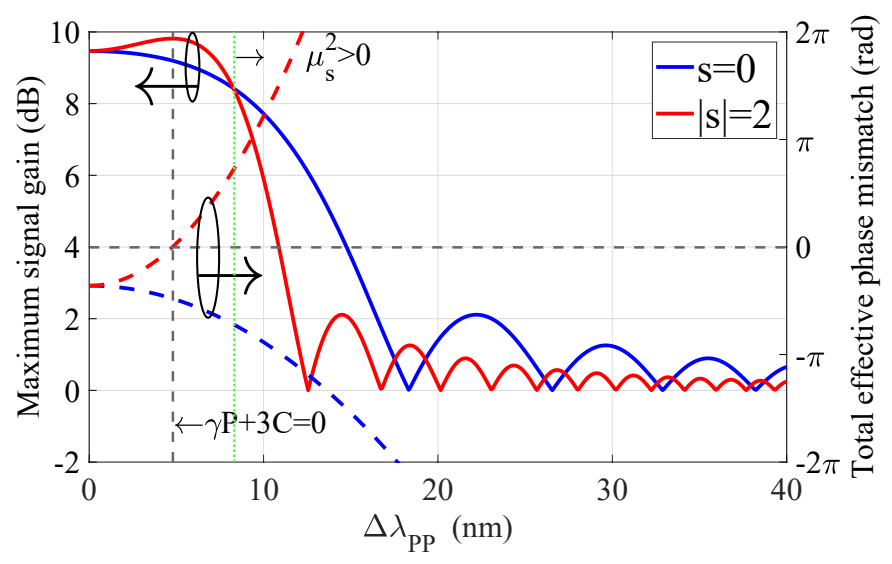

Fig. 5. Maximum PSA signal gain (left vertical axis) and total effective phase mismatch $\kappa_{t o t, 1-1 s-s} L$ (right vertical axis) versus $\Delta \lambda_{\text {PP }}$ for $s=0$ (blue solid lines) and $|s|=2$ (red dotted lines). Vertical grey dashed line: value of $\Delta \lambda_{\mathrm{PP}}$ for which $\gamma P+3 C=0$. The green dotted line separate the regions where $\mu_{s}$ is real and imaginary. Same parameters as in Fig. 4, except the non-zero input signal and idler powers.

Figure 5 shows that the gain spectrum is flatter for $s=0$ compared to $|s|=2$. This is due to the fact that for $|s|=2$, near $\Delta \lambda_{\mathrm{PP}}=5 \mathrm{~nm}$, the total effective phase mismatch $\kappa_{t o t, 1-12-2}=$ $-2(\gamma P+3 C) L \approx 0$ (see Subsection 2.B) under the strong pump approximation. It is indicated with a vertical grey dashed line in Fig. 5. This effective phase matching leads to a highly efficient amplification of the signal, and explains the maximum near $\Delta \lambda_{\mathrm{PP}}=5 \mathrm{~nm}$ in the gain spectrum. On the contrary, when $s=0$, the total effective phase mismatch is $\kappa_{t o t, 1-100}=-2(\gamma P-$ $C) L$. Since, in our case, $C$ is always negative in the anomalous dispersion region, $\kappa_{t o t, 1-100}$ can never be 0 . In fact, $\kappa_{t o t, 1-1 s-s}=$ $2\left(C\left(1-s^{2}\right)-\gamma P\right) L$ can only be 0 for $|s|>1$, i.e. when the signal is outside the two pumps (see Fig. 2).

\section{Stability of the Solution}

An important aspect of the solution for a system of ODEs is its stability. Since later we would like to analyze the solution stability for the analytical 7-wave model, first we consider the present case of the simpler 4-wave model.

We know that the stability of the system depends on the eigenvalues of the coefficient matrix of the homogeneous part of Eq. (18). The eigenvalues $\lambda_{ \pm}$are given by $\pm i \gamma P \mu_{s} . \gamma P \mu_{s}$ is often referred to as the parametric gain coefficient of the system. Note that $\mu_{S}$ can be either real or imaginary, depending on $F_{s}$. Therefore, when $\mu_{s}$ is real, the solution is elliptic or stable, however when $\mu_{s}$ is imaginary, the solution is hyperbolic or unstable (see Eq. (20)). Note that this stability is neutral stability and not asymptotic stability as our system does not incorporate damping. This can also be seen from Fig. 5 where on the right of the green dotted line $\mu_{s}^{2}>0$, i.e. $\mu_{s}$ is real. Thus the maximum 
PSA gain is lower compared to the left side of the line where $\mu_{S}$ is imaginary.

\section{ANALYTICAL 7-WAVE MODEL}

So far we have dealt with the well-established analytical 4-wave model, as it can be found in Ref. [12]. However, the special treatment of $|s|=3$ case is often overlooked in the standard literature. However, here, the introduction of the analytical 4wave model is motivated by the fact that we want to develop an analytical 7-wave model in terms of interactions between different 4-wave subsystems of the 7-wave system.

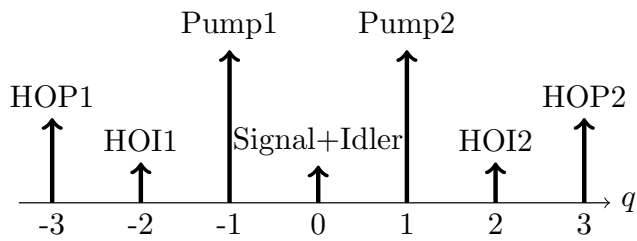

Fig. 6. Configuration of a 7-wave model with two pumps, degenerate signal and idler, high-order idlers (HOI1 and HOI2), and high-order pumps (HOP1 and HOP2).

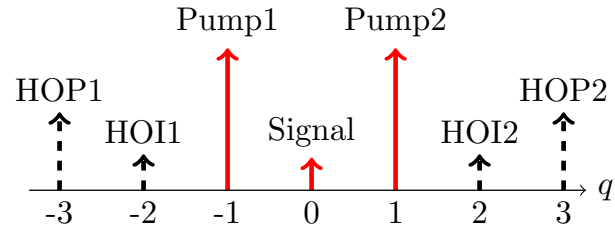

(a) Subsystem $1(s=0)$

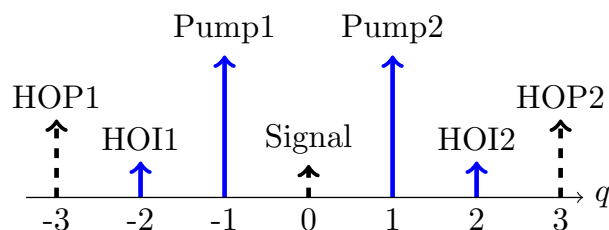

(b) Subsystem $2(|s|=2)$

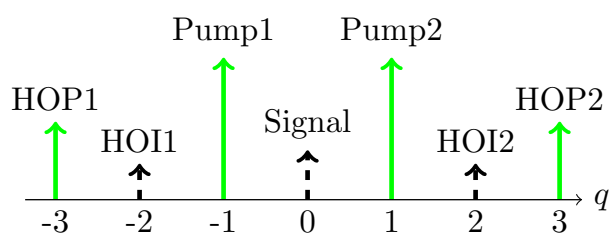

(c) Subsystem $3(|s|=3)$

Fig. 7. (a) Subsystem 1 ( $s=0$ in a 4-wave model) in red, (b) subsystem 2 ( $|s|=2$ in a 4-wave model) in blue and (c) subsystem $3(|s|=3$ in a 4-wave model) in green, used for describing a 7-wave model. Dashed arrows represent the waves that are not in the subsystem.

Similarly to the approach adopted for the analytical 4-wave model, in this section we apply the formalism developed in Section 2 to the case of 7 waves. The considered seven waves are : a degenerate signal and idler, two pumps, two HOIs and two HOPs (see Fig. 6). It is natural to expect the dynamics of the 7-wave model to be much more complicated than the 4-wave model simply because of the larger number of FWM terms in the coupled differential equations governing the propagation of the complex amplitudes $A_{j}$ 's. However, as we will see, implementing the strong pump approximation and analyzing the system in terms of interactions between its subsystems considerably simplifies the situation.

\section{A. Subsystems}

Although the general evolution equations of the seven waves, obtained from Eq. (3), are quite complicated, the strong pump approximation actually leads to a decoupling of the HOPs from the signal and HOIs. This provides a natural choice for breaking down the 7-wave system into three subsystems. These subsystems are three 4-wave systems with different values for the signal frequency parameter $s$. These subsystems are: i) the signal and the two pumps (subsystem 1, s=0, see Fig. 7 (a)), ii) the two HOIs and the two pumps (subsystem 2, $|s|=2$, see Fig. 7 (b)) and iii) the two HOPs and the two pumps (subsystem $3,|s|=3$, see Fig. 7 (c)). All the three subsystems share the two common pumps.

\section{B. HOP Evolution}

Let us first focus on subsystem 3, i.e., the two pumps with the HOPs. Similarly to the analytical 4-wave model (see Section $3)$, we write the equations for the complex amplitudes of the HOPs, i.e. $A_{ \pm 3}$, using Eq. (3) and invoking the strong pump approximation, leading to:

$$
\begin{aligned}
\frac{d}{d z}\left[\begin{array}{c}
A_{3} \\
A_{-3}{ }^{*}
\end{array}\right]=i \gamma P & {\left[\begin{array}{cc}
4 & 2 e^{i(6 \gamma P-16 C) z} \\
-2 e^{-i(6 \gamma P-16 C) z} & -4
\end{array}\right]\left[\begin{array}{c}
A_{3} \\
A_{-3} *
\end{array}\right] } \\
+ & {\left[\begin{array}{c}
i \gamma P^{\frac{3}{2}} e^{i(3 \gamma P-8 C) z} \\
-i \gamma P^{\frac{3}{2}} e^{-i(3 \gamma P-8 C) z}
\end{array}\right] . }
\end{aligned}
$$

The above equation is nothing but Eq. (15) with $|s|=3$. Its solution has already been discussed in Section 3 and given by Eq. (20) with $|s|=3$. Therefore, the strong pump approximation leads to a complete decoupling of subsystem 3 from subsystems 1 and 2, meaning the HOPs evolve independently from the signal and the HOIs.

\section{Coupled Signal and HOI Evolution}

Unlike subsystem 3, that can be solved using the 4-wave model, the dynamics of subsystems 1 and 2 are more complex. Indeed, the FWM processes involving the signal and HOIs, keep subsystems 1 and 2 strongly coupled with each other. Proceeding as before, with the strong pump approximation and using Eq. (3), we write the coupled signal and HOIs equations of evolution as:

$$
\frac{d}{d z}\left[\begin{array}{c}
A_{0} \\
A_{0}{ }^{*} \\
A_{2} \\
A_{2}{ }^{*} \\
A_{-2} \\
A_{-2}{ }^{*}
\end{array}\right]=i \gamma P\left[\begin{array}{cccccc}
4 & 2 e^{i(6 \gamma P+2 C) z} & 2 e^{i 4 C z} & e^{i(6 \gamma P-2 C) z} & 2 e^{i 4 C z} & e^{i(6 \gamma P-2 C)} \\
-2 e^{-i(6 \gamma P+2 C) z} & -4 & -e^{-i(6 \gamma P-2 C) z} & -2 e^{-i 4 C z} & -e^{-i(6 \gamma P-2 C) z} & -2 e^{-i 4 C z} \\
2 e^{-i 4 C z} & e^{i(6 \gamma P-2 C) z} & 4 & 0 & 0 & 2 e^{i(6 \gamma P-6 C) z} \\
-e^{-i(6 \gamma P-2 C) z} & -2 e^{i 4 C z} & 0 & -4 & -2 e^{-i(6 \gamma P-6 C) z} & 0 \\
2 e^{-i 4 C z} & e^{i(6 \gamma P-2 C) z} & 0 & 2 e^{i(6 \gamma P-6 C) z} & 4 & 0 \\
-e^{-i(6 \gamma P-2 C) z} & -2 e^{i 4 C z} & -2 e^{-i(6 \gamma P-6 C) z} & 0 & 0 & -4
\end{array}\right]\left[\begin{array}{c}
A_{0} \\
A_{0}^{*} \\
A_{2} \\
A_{2}^{*} \\
A_{-2} \\
A_{-2}{ }^{*}
\end{array}\right] .
$$


Equation (26) is a system of linear non-autonomous coupled ODEs. In particular, it takes into account several FWM frequency conversion processes, such as process $p_{1-2-10}$ involving waves $-2,-1,0$, and 1 and process $p_{-1201}$ coupling waves labeled $-1,0$, 1 , and 2 . These processes have to be taken into account here because of the particular frequency allocation of the different waves. These processes explain why $A_{0}$ is coupled to $A_{2}$ and $A_{-2}$.

A general approach to deal with such ODEs is to write the solution in terms of a matrix exponential and evaluating the exponential using the Magnus expansion method [42]. Other examples of this kind of dynamics arise in Dyson series in scattering theory [43] and Bloch equations used to describe electron spin resonances [44]. Nevertheless, a matrix exponential approach can often be cumbersome to implement. To avoid complication, here we use the simpler eigenvalue method to solve this system. But the coefficient matrix in Eq. (26) has a dimension $6 \times 6$ and also depends on $z$. Even if we eliminate the $z$-dependence, finding the eigenvalues of a $6 \times 6$ matrix requires finding the roots of a 6 th degree polynomial. Unfortunately, there is no closed form expression for the roots of a 6th degree polynomial. Therefore it is necessary to reduce the dimension of the coefficient matrix. It is worth noticing, by the way, that the case of degenerate signal and idlers (two pumps, signal and two HOIs) is more complicated to analyze than the non-degenerate signal and idler case (two pumps, signal, idler and two HOIs), for which the dimension of the coefficient matrix is $4 \times 4$ [26].

To reduce the dimension of the problem, we define the sideband imbalance $\delta_{2}(z)$ as:

$$
\delta_{2}(z)=A_{-2}(z)-A_{2}(z)
$$

The evolution equation for $\delta_{2}$ reads:

$$
\frac{d \delta_{2}}{d z}=\frac{d A_{-2}}{d z}-\frac{d A_{2}}{d z}=i \gamma P\left(4 \delta_{2}-2 e^{i(6 \gamma P-6 C) z} \delta_{2}^{*}\right)
$$

Note that $\delta_{2}$ does not depend on $A_{0}$,which means that an explicit solution of $\delta_{2}$ is obtainable. Therefore we can now express $A_{2}$ and $A_{2}{ }^{*}$ in terms of $\delta_{2}$ and reduce the dimension of the matrix in Eq. (26) to $4 \times 4$. After making a transformation of variables as shown in Eq. (17) (with $s=0,2,-2$ ), we obtain the following equation of evolution of the system:

$$
\begin{aligned}
\frac{d}{d z}\left[\begin{array}{c}
B_{0} \\
B_{0}{ }^{*} \\
B_{-2} \\
B_{-2}{ }^{*}
\end{array}\right]=i \gamma P\left[\begin{array}{cccc}
1-F_{0} & 2 & 4 & 2 \\
-2 & -\left(1-F_{0}\right) & -2 & -4 \\
2 & 1 & 1-F_{2} & 2 \\
-1 & -2 & -2 & -\left(1-F_{2}\right)
\end{array}\right]\left[\begin{array}{c}
B_{0} \\
B_{0}{ }^{*} \\
B_{-2} \\
B_{-2}{ }^{*}
\end{array}\right] \\
+i \gamma P\left[\begin{array}{c}
-2 \tilde{\delta}_{2}-\tilde{\delta}_{2}^{*} \\
2 \tilde{\delta}_{2}^{*}+\tilde{\delta}_{2} \\
-2 \tilde{\delta}^{*} \\
2 \tilde{\delta_{2}}
\end{array}\right],
\end{aligned}
$$

where $\tilde{\delta_{2}}=B_{-2}-B_{2}$. The evolution of $\tilde{\delta_{2}}$ is obtained by solving Eq. (28) and is given by:

$$
\begin{aligned}
\tilde{\delta_{2}}(z)= & \left(\frac{-i \eta_{2} \sin \left(\gamma P \mu_{2} z\right)}{\mu_{2}}+\cos \left(\gamma P \mu_{2} z\right)\right) \tilde{\delta}_{2}(0) \\
& -\left(\frac{2 i \sin \left(\gamma P \mu_{2} z\right)}{\mu_{2}}\right) \tilde{\delta}_{2}{ }^{*}(0) .
\end{aligned}
$$

Equation (29) has the form $d \mathbf{B} / d z=\mathbf{M B}+\mathbf{N}(z)$. Considering the homogeneous part of the equation, the coupling of subsystems 1 and 2 can be described by breaking $\mathbf{M}$ into four $2 \times 2$ block matrices $\mathbf{M}_{11}, \mathbf{M}_{12}, \mathbf{M}_{\mathbf{2 1}}$, and $\mathbf{M}_{\mathbf{2 2}}$ given as :

$$
\begin{aligned}
& \mathbf{M}_{11}=\operatorname{i\gamma } P\left[\begin{array}{cc}
1-F_{0} & 2 \\
-2 & -\left(1-F_{0}\right)
\end{array}\right], \quad \mathbf{M}_{12}=i \gamma P\left[\begin{array}{cc}
4 & 2 \\
-2 & -4
\end{array}\right]=2 \mathbf{M}_{21}, \\
& \mathbf{M}_{21}=\operatorname{i\gamma } P\left[\begin{array}{cc}
2 & 1 \\
-1 & -2
\end{array}\right], \quad \mathbf{M}_{22}=i \gamma P\left[\begin{array}{cc}
1-F_{2} & 2 \\
-2 & -\left(1-F_{2}\right)
\end{array}\right] .
\end{aligned}
$$

The diagonal blocks $\mathbf{M}_{11}$ and $\mathbf{M}_{\mathbf{2 2}}$ are the coefficient matrices for a 4-wave model with $s=0$ and $|s|=2$ respectively. On the other hand, the off-diagonal blocks $\mathbf{M}_{12}$ and $\mathbf{M}_{\mathbf{2 1}}$ describe how the two subsystems interact with each other.

The solution of Eq. (29) can be expressed as :

$$
\mathbf{B}(z)=\mathbf{F}(z) \mathbf{F}(0)^{-1} \mathbf{B}(0)+\int_{0}^{z} \mathbf{F}(z) \mathbf{F}(s)^{-1} \mathbf{N}(s) d s,
$$

where $\mathbf{F}(z)$ is the fundamental matrix of $\mathbf{M}$. With the aid of Mathematica, we compute the analytical solution to be:

$$
\begin{aligned}
& B_{0}(z)=B_{0}(0)\left(\cos \left(\gamma P v_{0} z\right)+\frac{i\left(\zeta^{2}+v_{0}^{2}\right)}{2 \zeta v_{0}} \sin \left(\gamma P v_{0} z\right)-\frac{3 i}{\zeta v_{2}} \sin \left(\gamma P v_{2} z\right)\right)+B_{0}^{*}(0)\left(\frac{i\left(-\zeta^{2}+v_{0}^{2}\right)}{2 \zeta v_{0}} \sin \left(\gamma P v_{0} z\right)-\frac{3 i}{\zeta v_{2}} \sin \left(\gamma P v_{2} z\right)\right)+B b_{-2}(0)\left(\frac{1}{2 \zeta} \cos \left(\gamma P v_{0} z\right)\right. \\
& \left.-\frac{1}{2 \zeta} \cos \left(\gamma P v_{2} z\right)+\frac{i}{2 v_{0}} \sin \left(\gamma P v_{0} z\right)+\frac{3 i}{2 v_{2}} \sin \left(\gamma P v_{2} z\right)\right)+B_{-2}^{*}(0)\left(-\frac{1}{2 \zeta} \cos \left(\gamma P v_{0} z\right)+\frac{1}{2 \zeta} \cos \left(\gamma P v_{2} z\right)-\frac{i}{2 v_{0}} \sin \left(\gamma P v_{0} z\right)+\frac{3 i}{2 v_{2}} \sin \left(\gamma P v_{2} z\right)\right)+ \\
& B_{2}(0)\left(\frac{1}{2 \zeta} \cos \left(\gamma P v_{0} z\right)-\frac{1}{2 \zeta} \cos \left(\gamma P v_{2} z\right)+\frac{i}{2 v_{0}} \sin \left(\gamma P v_{0} z\right)+\frac{3 i}{2 v_{2}} \sin \left(\gamma P v_{2} z\right)\right) \\
& +B_{2}^{*}(0)\left(-\frac{1}{2 \zeta} \cos \left(\gamma P v_{0} z\right)+\frac{1}{2 \zeta} \cos \left(\gamma P v_{2} z\right)-\frac{i}{2 v_{0}} \sin \left(\gamma P v_{0} z\right)+\frac{3 i}{2 v_{2}} \sin \left(\gamma P v_{2} z\right)\right)
\end{aligned}
$$

$$
\begin{aligned}
& B_{-2}(z)=B_{0}(0)\left(\frac{1}{2 \zeta} \cos \left(\gamma P v_{0} z\right)-\frac{1}{2 \zeta} \cos \left(\gamma P v_{2} z\right)+\frac{i}{2 v_{0}} \sin \left(\gamma P v_{0} z\right)+\frac{3 i}{2 v_{2}} \sin \left(\gamma P v_{2} z\right)\right)+B_{0}^{*}(0)\left(\frac{1}{2 \zeta} \cos \left(\gamma P v_{0} z\right)-\frac{1}{2 \zeta} \cos \left(\gamma P v_{2} z\right)-\frac{i}{2 v_{0}} \sin \left(\gamma P v_{0} z\right)+\right. \\
& \left.\frac{3 i}{2 v_{2}} \sin \left(\gamma P v_{2} z\right)\right)+B_{-2}(0)\left(\frac{1}{2} \cos \left(\gamma P v_{2} z\right)+\frac{1}{2} \cos \left(\gamma P \mu_{2} z\right)+\frac{i}{2 \zeta v_{0}} \sin \left(\gamma P v_{0} z\right)-\frac{i\left(3 \zeta^{2}+2 \zeta+1\right)}{2 \zeta v_{2}} \sin \left(\gamma P v_{2} z\right)-\frac{i(2+3 \zeta)}{2 \mu_{2}} \sin \left(\gamma P \mu_{2} z\right)\right) \\
& +B_{-2}^{*}(0)\left(-\frac{i}{2 \zeta v_{0}} \sin \left(\gamma P v_{0} z\right)+\frac{i(2 \zeta+1)}{2 \zeta v_{2}} \sin \left(\gamma P v_{2} z\right)-\frac{i}{\mu_{2}} \sin \left(\gamma P \mu_{2} z\right)\right)+B_{2}(0)\left(\frac{1}{2} \cos \left(\gamma P v_{2} z\right)-\frac{1}{2} \cos \left(\gamma P \mu_{2} z\right)+\frac{i}{2 \zeta v_{0}} \sin \left(\gamma P v_{0} z\right)\right. \\
& \left.-\frac{i\left(3 \zeta^{2}+2 \zeta+1\right)}{2 \zeta v_{2}} \sin \left(\gamma P v_{2} z\right)+\frac{i(2+3 \zeta)}{2 \mu_{2}} \sin \left(\gamma P \mu_{2} z\right)\right)+B_{2}^{*}(0)\left(-\frac{i}{2 \zeta v_{0}} \sin \left(\gamma P v_{0} z\right)+\frac{i(2 \zeta+1)}{2 \zeta v_{2}} \sin \left(\gamma P v_{2} z\right)+\frac{i}{\mu_{2}} \sin \left(\gamma P \mu_{2} z\right)\right)
\end{aligned}
$$


where $\zeta$ and $v_{s}$ (with $s=0,2$ ) are given by :

$$
\zeta=-1-F_{0}, v_{s}=\sqrt{3-2 F_{s}+F_{s}^{2}} .
$$

For brevity here we have not provided the expression for $B_{2}$ as it can be easily computed using $B_{2}=B_{-2}-\tilde{\delta_{2}}$. Note that $v_{\mathcal{S}}$ (unlike $\mu_{s}$ ), is always a real quantity. $v_{s}$ and $\mu_{s}$ are related by :

$$
v_{s}^{2}=\mu_{s}^{2}+6
$$

One important consequence of the above signal solution is that, while for the analytical 3-wave model, the modulation instability of the signal originates from the solution having hyperbolic terms (when $\mu_{0}$ is imaginary), in contrast, for the analytical 7wave model, the signal modulation instability is a consequence of the larger value of the parametric gain coefficient $v_{0}$ compared to $\mu_{0}$, mediated through the coupling of the HOI modes to the signal. We also point out the fact that, although the signal solution from the analytical 7-wave model only contains sinusoidal terms, the HOIs can have both sinusoidal and hyperbolic terms when $\mu_{2}$ is imaginary.

\section{Maximum Signal Gain}

In this subsection we consider the case where only the two pumps and the signal are injected at the fiber input. This situation generalizes the one described in the 3-wave model by taking the generated HOIs into account. In order to compare the results of the analytical 3- and 7-wave models, Fig. 8 (a) shows the evolution of the maximum PSA signal gain (with respect to the input signal phase) as a function of $\Delta \lambda_{\mathrm{PP}}$ obtained in both cases. Figure $8(\mathrm{~b})$ shows the corresponding total effective phase mismatches $\kappa_{t o t, 1-100} L$ (blue solid), $\kappa_{t o t,-1-1-20} L$ (red dotted) and $\kappa_{t o t, 1-2-10} L$ (green dot-dashed), corresponding to the FWM processes $p_{1-100}, p_{-1-1-20}$ and $p_{1-2-10}$ respectively (see Fig. 9, as a function of $\Delta \lambda_{\mathrm{PP}}$. The total effective phase mismatches are calculated as discussed in Subsection 2.B with the strong pump approximation. In the analytical 3-wave model only the process $p_{1-100}$ is considered, whereas in the analytical 7-wave model all the three processes $\left(p_{1-100}, p_{-1-1-20}\right.$, and $\left.p_{1-2-10}\right)$ are considered.

Figure 8(a) shows that for small values of $\Delta \lambda_{\mathrm{PP}}(<5 \mathrm{~nm})$, the two models show a large difference in maximum signal gain. This is explained by the fact that when $\Delta \lambda_{\mathrm{PP}}$ is small, the FWM process $p_{1-2-10}$ is highly phase matched (see green dotdashed curve in Fig. 8(b)) and thus plays a significant role in determining the maximum PSA signal gain. Since this process is not considered in the analytical 3-wave model, we find a large discrepancy between the two models.

Near $\Delta \lambda_{\mathrm{PP}}=8 \mathrm{~nm}$, the process $p_{-1-1-20}$ gets perfectly phase matched (see red dotted curve in Fig. $8(\mathrm{~b})$ ), which is also not accounted for in the analytical 3-wave model. This process leads to an efficient amplification of the signal and is responsible for the large gain peak for the analytical 7-wave model in Fig. 8 (a) (red dotted curve) around $\Delta \lambda_{\mathrm{PP}}=8 \mathrm{~nm}$, which is absent for the analytical 3-wave model (blue solid curve). The slight mismatch between the gain peak and the perfect phase matching region of process $p_{-1-1-20}$ is due to the presence of the two other processes $p_{1-100}$ and $p_{1-2-10}$, which are not very efficient but not negligible.

As $\Delta \lambda_{\mathrm{PP}}$ increases, all the FWM processes start becoming phase mismatched and lose their efficiency. Thus, for $\Delta \lambda_{\mathrm{PP}}>$
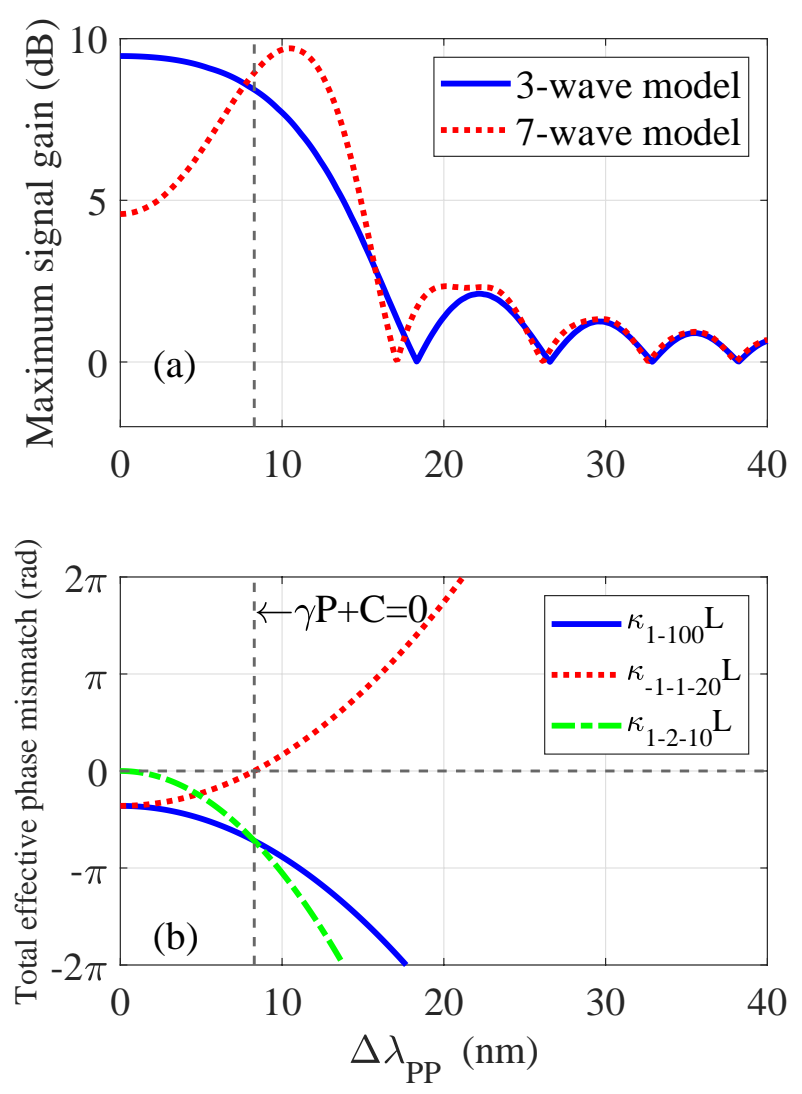

Fig. 8. (a) Maximum signal gain for analytical 3-wave (full blue line) and 7-wave (dotted red line) models versus $\Delta \lambda_{\mathrm{PP}}$. (b) Corresponding total effective phase mismatches $\kappa_{t o t, 1-100}$ (full blue line), $\kappa_{t o t,-1-1-20}$ (dotted red line) and $\kappa_{t o t, 1-2-10}$ (dot-dashed green line) for FWM processes $p_{1-100}, p_{-1-1-20}$ and $p_{1-2-10}$ respectively (see Fig. 9). Vertical grey dashed line: value of $\Delta \lambda_{\text {PP }}$ where $\gamma P+C=0$. Same parameters as in Fig. 4 .

$30 \mathrm{~nm}$, the HOIs remain negligibly weak. In such a case, the analytical 3- and 7-wave models predict the same maximum signal gain (see Fig. 8 (a)).

\section{E. Comparison of the Analytical 3- and 7-Wave Models with the Numerical 7-Wave Model}

In our approach to develop the analytical 7-wave model, we only considered the terms that contain at least two pump amplitudes in Eq. (3), allowing us to obtain a linear system of coupled differential equations. This limits the validity of our model. With the increase of either the pump power, the length, or the nonlinear coefficient of the fiber, the FOPA starts behaving more and more nonlinearly. Thus when the nonlinear phase $\gamma P L$ becomes strong, the generated sidebands, i.e., $A_{ \pm 2}$ and $A_{ \pm 3}$, play an important role in the dynamics of the FOPA. In such cases the developed analytical approach should become inapplicable.

In contrast to an analytical approach, using a numerical approach allows one to solve the set of seven coupled nonlinear differential equations, without need of the strong pump approximation [35]. The complete set of coupled equations in this case can be found in Ref. [45].

Thus, in order to validate our approach, we compare the dependence of the maximum PSA signal gain (obtained by optimizing the input signal phase) on $\Delta \lambda_{\mathrm{PP}}$, calculated using the analytical 3- and 7-wave models and the numerical 7-wave model, 


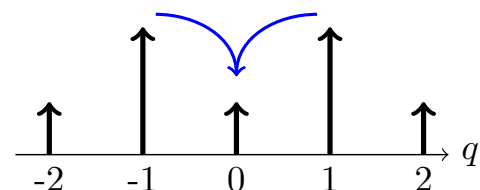

(a) $p_{1-100}(3 \mathrm{wm}, 7 \mathrm{wm})$

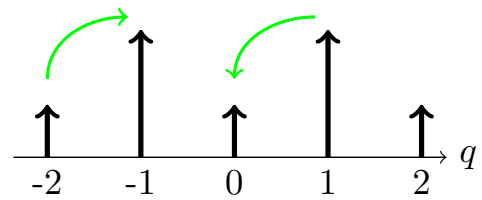

(b) $p_{1-2-10}(7 \mathrm{wm})$

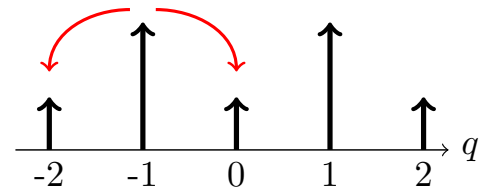

(c) $p_{-1-1-20}(7 \mathrm{wm})$

Fig. 9. The different FWM processes considered in the analytical 3-wave model (only (a)) and analytical 7-wave model $\left((\mathrm{a}),(\mathrm{b})\right.$ and (c)). The process labelled $p_{m n p j}$ involves waves at frequencies $\omega_{m}, \omega_{n}, \omega_{p}$, and $\omega_{j}$.
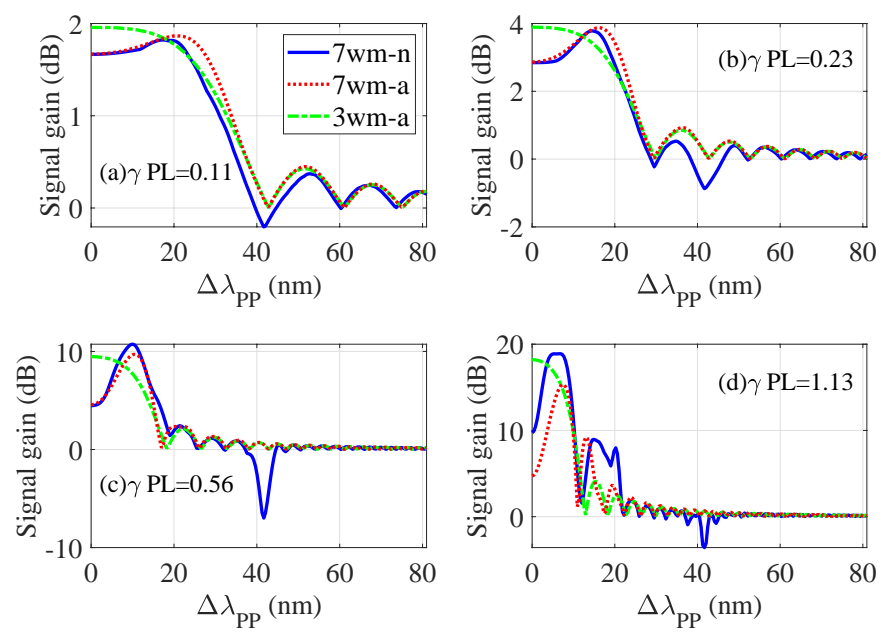

Fig. 10. Maximum signal gain versus $\Delta \lambda_{\mathrm{PP}}$ for the the 3-wave model (green dot-dashed line), the analytical 7-wave model (red dotted line), and numerical 7-wave model (blue solid line). (a) $\gamma P L=0.11$, (b) $\gamma P L=0.23$, (c) $\gamma P L=0.56$, and (d) $\gamma P L=1.13$. Same parameters as in Fig. 4 .

for different values of $\gamma P L$, when the signal is in the anomalous $\left(\lambda_{0}=1557.5 \mathrm{~nm}\right.$, Fig. 10$)$ and normal $\left(\lambda_{0}=1537.5 \mathrm{~nm}\right.$, Fig. 11$)$ dispersion regions. Note that here only the two pumps and the signal are launched at the fiber input. For the numerical 7-wave model, we neglected the fiber attenuation, considered a 4th order approximation for the linear phase mismatch coefficients, and took into account all the FWM terms obtained from Eq. (3).

From Fig. 10, we conclude that all the three models predict approximately the same signal gain for large pump-pump wavelength separations $\left(\Delta \lambda_{\mathrm{PP}}>60 \mathrm{~nm}\right)$. This is due to the fact that, when $\Delta \lambda_{\mathrm{PP}}$ is large, all the FWM processes get highly phase mismatched, and thus the high-order waves are not generated efficiently. Therefore the only process that dominates is the main
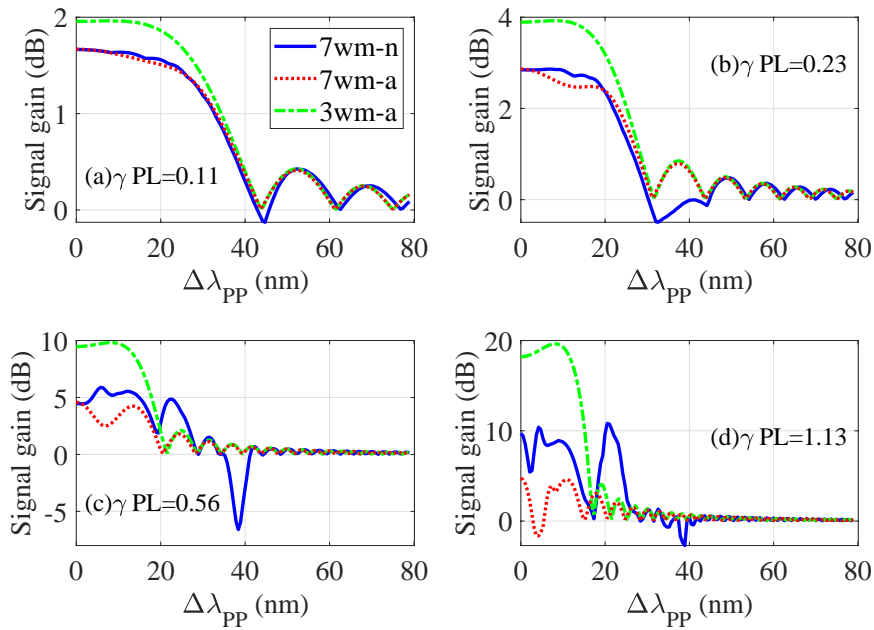

Fig. 11. Same as Fig. 10 for the normal dispersion region $\left(\lambda_{0}=\right.$ $1537.5 \mathrm{~nm})$.

one considered in the 3-wave model involving the signal and the two pumps.

One can also see that, for $\Delta \lambda_{\mathrm{PP}}<10 \mathrm{~nm}$ and $\gamma P L<0.6 \mathrm{rad}$ (see Figs. 10(a,b,c)), the analytical 3-wave model deviates significantly from the 7-wave numerical model, whereas the analytical 7-wave model approximately agrees with the numerical 7-wave model. However, when $\gamma P L>0.6$ rad (see Fig. $10(\mathrm{~d})$ ) the analytical 7-wave model predicts a different maximum PSA gain compared to the numerical 7-wave model even when $\Delta \lambda_{\mathrm{PP}}<10$ $\mathrm{nm}$. This is caused by the fact that for $\gamma P L>0.6 \mathrm{rad}$, the HOPs become strong enough to deplete the pump powers significantly, and hence the strong pump approximation breaks down.

Similarly to the preceding discussion, Fig. 11 shows that for $\Delta \lambda_{\mathrm{PP}}<40 \mathrm{~nm}$, although the analytical 3-wave model always deviates significantly from the numerical 7 -wave model, the analytical and numerical 7-wave models agree satisfactorily as long as $\gamma P L<0.3 \mathrm{rad}$. However, as $\gamma P L$ increases, for example for $\gamma P L=0.56 \mathrm{rad}$ (Fig. 11 (c)) and $\gamma P L=1.13 \mathrm{rad}$ (Fig. $11(\mathrm{~d})$ ), the analytical and numerical 7-wave models disagree for $\Delta \lambda_{\mathrm{PP}}<20 \mathrm{~nm}$. Nevertheless, the case of the signal being in the normal dispersion region is of reduced interest, since the maximum attainable signal gain in this region (in the considered range of $\Delta \lambda_{\mathrm{PP}}$ ) is much smaller than that for the anomalous dispersion region.

From the above comparison between the analytical and numerical 7-wave models, one can conclude that the analytical 7-wave model provides a satisfactorily accurate description of the signal wave evolution in the anomalous dispersion regime, when $\Delta \lambda_{\mathrm{PP}}<15 \mathrm{~nm}$ and $\gamma P L<0.6$, as under such conditions the FWM processes $p_{1-100}, p_{-1-1-20}$ and $p_{1-2-10}$ are the only dominant processes determining the system dynamics.

\section{F. Injection of the HOls at the Fiber Input}

Using the solution of the analytical 7-wave model, in Fig.12 we plot the maximum PSA signal gain (optimizing the input phases of the signal and the HOIs) as a function of $\Delta \lambda_{\text {PP }}$ for different values of $r_{-2}$ and $r_{2}$, where $r_{-2}$ and $r_{2}$ are the ratios of powers of the HOIs to the power of the signal at the input of the fiber. These ratios $r_{2}$ and $r_{-2}$ are thus expressed as :

$$
r_{-2}=\frac{\left|B_{-2}(0)\right|^{2}}{\left|B_{0}(0)\right|^{2}}, \quad r_{2}=\frac{\left|B_{2}(0)\right|^{2}}{\left|B_{0}(0)\right|^{2}} .
$$




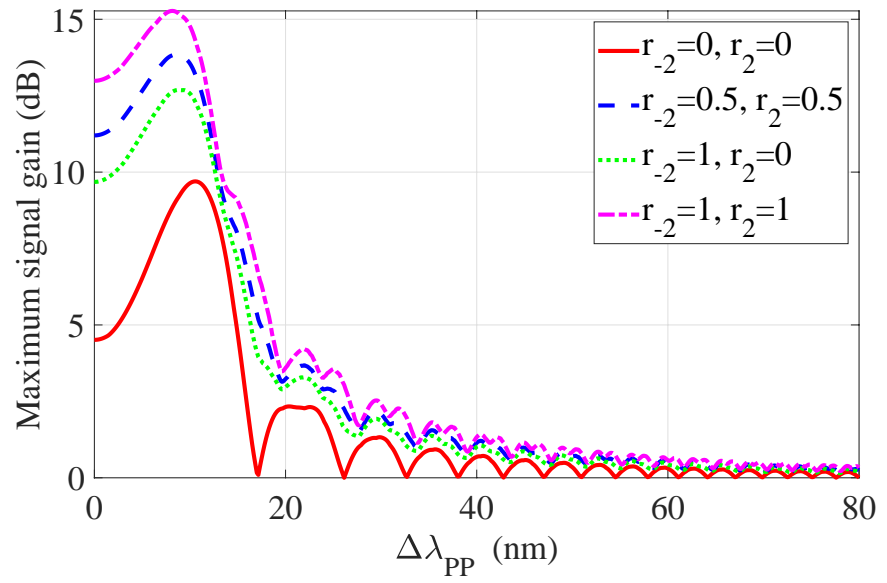

Fig. 12. Maximum PSA signal gain versus $\Delta \lambda_{\mathrm{PP}}$ using the analytical 7-wave model for different values of $r_{-2}$ and $r_{2}$. Input signal power $P_{0}(0)=-20 \mathrm{dBm}$; signal wavelength $\lambda_{0}=1557.5 \mathrm{~nm}$. Other parameters: same as in Fig. 4 .

We see from Fig. 12 that injecting the HOIs at the input of the fiber leads to a strong increase of the maximum signal gain (about $5 \mathrm{~dB}$ increase with $\gamma P L=0.5$, and $r_{-2}=r_{2}=1$ ) compared to the situation when HOIs are not injected $\left(r_{-2}=r_{2}=0\right)$. Also, injection of equal input powers for the HOIs is a more effective strategy for the signal gain enhancement, compared to unequal input HOI powers as can be seen by comparing the green dotted curve $\left(r_{-2}=1\right.$ and $\left.r_{2}=0\right)$ with the blue dashed curve $\left(r_{-2}=0.5\right.$ and $\left.r_{2}=0.5\right)$ in Fig. 12 . This is also reminiscent of the fact that a PSA redistributes the energy from the pumps in a way to equalize the signal and the idler(s) [46]. Therefore when the HOIs are unequal, a large part of the pump power is invested in amplifying the weaker HOI. Such an effect is probably also connected to the initial stage of the Fermi-Pasta-Ulam recurrence phenomenon in a coupled oscillator system, where the energy of one oscillating mode gets redistributed to other modes with lower initial energies [47]. These results are also interesting in the context of high-gain fiber PSA design. However, locking the phases of the five waves (two pumps, signal and two HOIs) might be a challenge in practice.

\section{DISCUSSION AND CONCLUSION}

In this section we first outline an important limitation of the developed analytical 7-wave model with respect to the calculation of linear phase mismatch coefficients. Then we summarize the important results of this investigation.

\section{A. Validity of Second Order Approximation in Linear Phase Mismatch Coefficient Calculation}

In Subsection 2.A, the linear phase mismatch coefficients were calculated considering a second order approximation, which can be a source of error. In the literature, Liu considered a fifth order approach to calculate the coefficients, for $\Delta \lambda_{\mathrm{PP}}<50 \mathrm{~nm}$ [48]. However Marhic et al. in Ref. [49] considered a fourth order approach for a similar situation. Therefore it is important to investigate the validity of this second order approximation to calculate the $\Delta \beta_{m n p j}$ 's. To this aim, we plot in Fig. 13 the evolution of $\Delta \beta_{1-100} L$ (blue lines), $\Delta \beta_{1-2-10} L$ (green lines), and $\Delta \beta_{-1-1-20} L$ (red lines), considering second (thick lines) and fourth (thin lines) order Taylor series expansion of $\beta(\omega)$, as a function of $\Delta \lambda_{\mathrm{PP}}$. The parameters are those of our standard HNLF of length $L=500 \mathrm{~m}$.

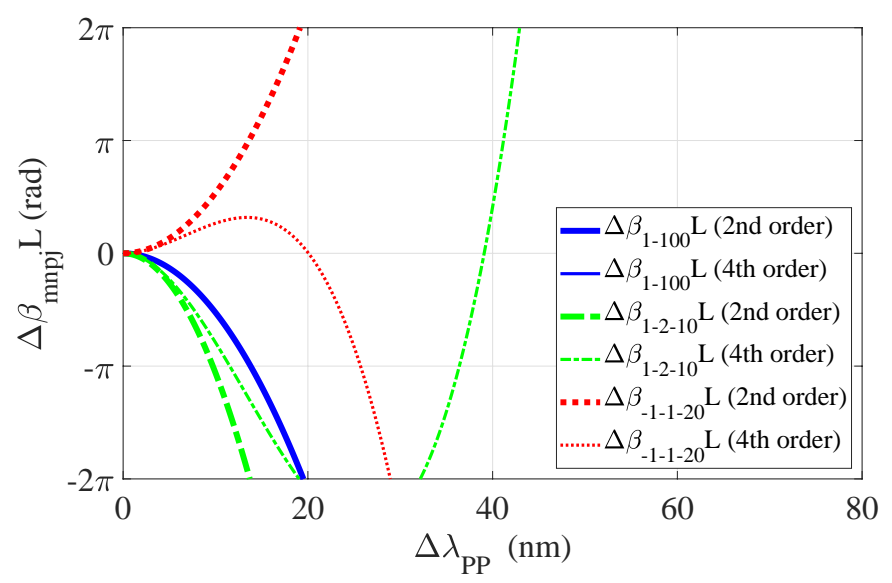

Fig. 13. Evolution of $\Delta \beta_{m n p j} L$ versus $\Delta \lambda_{\mathrm{PP}}$ for three processes and comparing second-order (thick lines) and fourth-order (thin lines) Taylor series expansion of $\beta(\omega) . L=500 \mathrm{~m}$. Other parameters: same as in Fig. 4.

Figure 13 shows that for small values of $\Delta \lambda_{\mathrm{PP}}(<10 \mathrm{~nm})$, the error accumulated by performing a second order expansion is not significant $(<\pi / 4)$. However, for $\Delta \beta_{1-2-10} L$ and $\Delta \beta_{-1-1-20} L$ the error increases rapidly with $\Delta \lambda_{\mathrm{PP}}$. Since near $\Delta \lambda_{\mathrm{PP}}=10 \mathrm{~nm}$, the only dominant process is $p_{-1-1-20}$ (see Fig. $8(\mathrm{~b})$ ), we need not consider the error from $p_{1-2-10}$. We also note here that the deviation between the second and fourth order approaches is 0 for $\Delta \beta_{1-100} L$, which corresponds to the FWM process considered in the analytical 3-wave model, due to the choice of $\omega_{c}=\omega_{0}$.

Figure 13 also shows that the 4 th order calculation predicts that the processes $p_{-1-1-20}$ and $p_{1-2-10}$ are supposed to become perfectly phase matched around $\Delta \lambda_{\mathrm{PP}}=20 \mathrm{~nm}$ and $\Delta \lambda_{\mathrm{PP}}=40 \mathrm{~nm}$, respectively. This implies that the PSA dynamics in these regions will not be correctly captured by the developed analytical 7-wave model. This explains the large dip in the gain spectrum around $\Delta \lambda_{\mathrm{PP}}=40 \mathrm{~nm}$ seen in Figs. 10 (c) and (d) (blue solid line). It is due to occurrence of the FWM process $p_{1-210}$, which converts a signal and a pump 1 photon into a HOI1 and a pump 2 photon [35]. Also, the hump in the gain spectrum around $\Delta \lambda_{\mathrm{PP}}=20 \mathrm{~nm}$ in Fig. 10 (d) (blue solid line) is due to the efficiency of $p_{-1-1-20}$, which pumps photons into the signal.

\section{B. Conclusion}

In conclusion, we derived a full analytical solution for the evolution of the slowly varying complex amplitudes of seven CW waves with two strong pumps propagating through a nonlinear fiber. This analytical 7 -wave model provides a more accurate description of the dynamics of a degenerate dual-pump PSA compared to the analytical 3-wave model. In particular, the coupling of the signal with the generated HOIs plays an important role in the signal amplification process. As opposed to the analytical 3-wave model, that explains the signal modulation instability in terms of hyperbolic solutions, the analytical 7-wave model provides an interpretation of the signal instability in terms of the parametric gain coefficient that gets enhanced by the coupling with the HOIs. We also found that such a coupling can be utilized to enhance the PSA gain by launching the HOIs at the fiber input with optimized phases. Such a knowledge is 
relevant for designing high-gain PSAs. The developed analytical model agreed satisfactorily with its numerical counterpart for $\Delta \lambda_{\mathrm{PP}}<15 \mathrm{~nm}$ and $\gamma P L<0.6$ rads, while working in the anomalous dispersion region. Apart from the strong pump approximation, the consideration of only the second order dispersion in the calculations is an important limitation of this model.

The results derived here provide key insights into the physics of propagation of multiple $\mathrm{CW}$ waves through a nonlinear optical medium. They also offer the possibility of analyzing FWM based all-optical regenerative $[50,51]$ and phase sensitive frequency converter [45] systems.

We envisage that this approach can be generalized to the case of an arbitrary number of CW waves. Such attempts might face the challenge of tackling pump depletion effects. Nevertheless, the approach of Chen and Snyder to incorporate pump depletion [20], together with the decoupling of the HOPs from the signal and HOIs in our model, might provide some insights into developing such generalized models.

Another perspective of this work deals with quantum noise. Indeed, we have recently shown that, using a semi-classical model, the quantum noise of a dual-pump degenerate PSA is mainly influenced by the HOIs rather than the HOPs [39]. Thus the derived expressions of the signal and HOI evolution can be utilized for calculations of quantum noise using a full quantum approach. Such investigations would reveal the role of dispersion of the fiber in influencing the quantum noise of a PSA.

Finally, we believe that this paper will stimulate further experimental work towards investigation of PSA systems with superior gain properties and opens new avenues for further research in nonlinear optics.

\section{APPENDIX}

This appendix provides an argument for the choice of variable transformation as given in Eq. (17). This transformation is primarily motivated by the perspective to get rid of the $z$ dependence of the coefficient matrix $\mathbf{M}(z)$ in Eq. (15).

Let us consider a system of non-autonomous homogeneous coupled system of ODEs as :

$$
\frac{d x_{k}}{d z}=\sum_{j=1}^{n} a_{k j} e^{i b_{k j} z} x_{j}
$$

where $k \in\{1,2 \ldots n\}$. We start from the ansatz :

$$
x_{k}=e^{i c_{k} z} y_{k}
$$

Plugging it back into Eq. (38), we have :

$$
i c_{k} e^{i c_{k} z} y_{k}+e^{i c_{k} z} \frac{d y_{k}}{d_{z}}=\sum_{j=1}^{n} a_{k j} e^{i\left(b_{k j}+c_{j}\right) z} y_{j}
$$

For the exponential terms to cancel out, we must satisfy :

$$
c_{k}-c_{j}=b_{k j}
$$

for all $k, j \in\{1,2 \ldots n\}$. We consider the coefficient matrix $\mathbf{M}(z)$ in Eq. (15) given by :

$$
\mathbf{M}(z)=i \gamma P\left[\begin{array}{cc}
4 & 2 e^{i\left(6 \gamma P+2 C\left(1-s^{2}\right)\right) z} \\
-2 e^{-i\left(6 \gamma P+2 C\left(1-s^{2}\right)\right) z} & -4
\end{array}\right] .
$$

Hence we get the only nontrivial equation for the $c_{k}$ 's as :

$$
c_{1}-c_{2}=6 \gamma P+2 C\left(1-s^{2}\right) .
$$

This equation has an infinite number of solutions. Without loss of generality, we choose $c_{2}=-c_{1}$. Thus we get :

$$
c_{1}=3 \gamma P+C\left(1-s^{2}\right) \text {. }
$$

Therefore the coordinate transformation to eliminate the $z$ dependence of the coefficient matrix in Eq. (15), is given by:

$$
A_{S}=e^{i\left(3 \gamma P+C\left(1-s^{2}\right)\right) z} B_{s}, \quad A_{-s}{ }^{*}=e^{-i\left(3 \gamma P+C\left(1-s^{2}\right)\right) z} B_{-s}{ }^{*} .
$$

\section{DISCLOSURES}

The authors declare no conflicts of interest.

\section{ACKNOWLEDGEMENT}

We thank Weilin Xie and Ihsan Fsaifes for their contributions to the early stages of this work.

\section{REFERENCES}

1. Z. Tong, C. Lundström, P. A. Andrekson, M. Karlsson, and A. Bogris, "Ultralow noise, broadband phase-sensitive optical amplifiers, and their applications," IEEE J. Sel. Top. Quantum Electron. 18, 1016-1032 (2011).

2. Z. Tong and S. Radic, "Low-noise optical amplification and signal processing in parametric devices," Adv. Opt. Photonics 5, 318-384 (2013).

3. M. E. Marhic, P. A. Andrekson, P. Petropoulos, S. Radic, C. Peucheret, and M. Jazayerifar, "Fiber optical parametric amplifiers in optical communication systems," Laser \& Photonics Rev. 9, 50-74 (2015).

4. M. Karlsson, "Transmission systems with low noise phase-sensitive parametric amplifiers," J. Light. Technol. 34, 1411-1423 (2015).

5. P. A. Andrekson and M. Karlsson, "Fiber-based phase-sensitive optical amplifiers and their applications," Adv. Opt. Photonics 12, 367-428 (2020).

6. O.-K. Lim, V. Grigoryan, M. Shin, and P. Kumar, "Ultra-low-noise inline fiber-optic phase-sensitive amplifier for analog optical signals," in $O p$ tical Fiber Communication Conference, (Optical Society of America, 2008), p. OML3.

7. D. Chatterjee, Y. Bouasria, W. Xie, T. Labidi, F. Goldfarb, I. Fsaifes, and F. Bretenaker, "Investigation of analog signal distortion introduced by a fiber phase sensitive amplifier," J. Opt. Soc. Am. B 37, 2405-2415 (2020).

8. P. Zhao, R. Kakarla, M. Karlsson, and P. A. Andrekson, "Enhanced analog-optical link performance with noiseless phase-sensitive fiber optical parametric amplifiers," Opt. Express 28, 23534-23544 (2020).

9. A. C. Sodré, N. Cañas-Estrada, D. F. Noque, R. M. Borges, S. A Melo, N. G. González, and J. C. Oliveira, "Photonic-assisted microwave amplification using four-wave mixing," IET Optoelectronics 10, 163-168 (2016).

10. R. Tang, P. S. Devgan, V. S. Grigoryan, P. Kumar, and M. Vasilyev, "In-line phase-sensitive amplification of multi-channel cw signals based on frequency nondegenerate four-wave-mixing in fiber," Opt. Express 16, 9046-9053 (2008)

11. S. L. Olsson, H. Eliasson, E. Astra, M. Karlsson, and P. A. Andrekson, "Long-haul optical transmission link using low-noise phase-sensitive amplifiers," Nat. Commun. 9, 1-7 (2018).

12. G. Agrawal, Nonlinear Fiber Optics Fifth Edition (Elsevier, 2013).

13. S. Radic and C. McKinstrie, "Two-pump fiber parametric amplifiers," Opt. Fiber Technol. 9, 7-23 (2003).

14. T. Torounidis, H. Sunnerud, P. O. Hedekvist, and P. A. Andrekson, "Amplification of wdm signals in fiber-based optical parametric amplifiers," IEEE Photonics Technol. Lett. 15, 1061-1063 (2003). 
15. J. Kakande, C. Lundström, P. A. Andrekson, Z. Tong, M. Karlsson, P. Petropoulos, F. Parmigiani, and D. J. Richardson, "Detailed characterization of a fiber-optic parametric amplifier in phase-sensitive and phase-insensitive operation," Opt. Express 18, 4130-4137 (2010).

16. C. Lundström, B. Corcoran, M. Karlsson, and P. A. Andrekson, "Phase and amplitude characteristics of a phase-sensitive amplifier operating in gain saturation," Opt. Express 20, 21400-21412 (2012).

17. T. Labidi, I. Fsaifes, W. Xie, D. Chatterjee, F. Goldfarb, and F. Bretenaker, "Phase evolution of the direct detection noise figure of a nondegenerate fiber phase-sensitive amplifier," Opt. Lett. 43, 4546-4549 (2018).

18. R. Stolen and J. Bjorkholm, "Parametric amplification and frequency conversion in optical fibers," IEEE J. Quantum Electron. 18, 1062-1072 (1982).

19. G. Cappellini and S. Trillo, "Third-order three-wave mixing in singlemode fibers: exact solutions and spatial instability effects," J. Opt. Soc. Am. B 8, 824-838 (1991).

20. Y. Chen and A. W. Snyder, "Four-photon parametric mixing in optical fibers: effect of pump depletion," Opt. Lett. 14, 87-89 (1989).

21. T. Hansson, A. Tonello, S. Trillo, and S. Wabnitz, "Modulation instability, four-wave mixing and their applications," in Shaping Light in Nonlinear Optical Fibers, S. Boscolo and C. Finot, eds. (John Wiley \& Sons, Ltd Chichester, UK, 2017), pp. 1-33.

22. S. Trillo, S. Wabnitz, and T. Kennedy, "Nonlinear dynamics of dualfrequency-pumped multiwave mixing in optical fibers," Phys. Rev. A 50, 1732 (1994).

23. J. R. Thompson and R. Roy, "Multiple four-wave mixing process in an optical fiber," Opt. Lett. 16, 557-559 (1991).

24. J. R. Thompson and R. Roy, "Nonlinear dynamics of multiple four-wave mixing processes in a single-mode fiber," Phys. Rev. A 43, 4987 (1991).

25. M. E. Marhic, K. K. Wong, and L. G. Kazovsky, "Fiber optical parametric amplifiers with linearly or circularly polarized waves," J. Opt. Soc. Am. B 20, 2425-2433 (2003).

26. A. Vedadi, M. E. Marhic, E. Lantz, H. Maillotte, and T. Sylvestre, "Investigation of gain ripple in two-pump fiber optical parametric amplifiers," Opt. Lett. 33, 2203-2205 (2008).

27. M. Marhic, A. Rieznik, and H. Fragnito, "Investigation of the gain spectrum near the pumps of two-pump fiber-optic parametric amplifiers," J. Opt. Soc. Am. B 25, 22-30 (2008).

28. M. E. Marhic, Fiber optical parametric amplifiers, oscillators and related devices (Cambridge university press, 2008)

29. T. Tanemura and K. Kikuchi, "Unified analysis of modulational instability induced by cross-phase modulation in optical fibers," J. Opt. Soc. Am. B 20, 2502-2514 (2003).

30. H. Pakarzadeh and A. Zakery, "Investigation of two-pump fiber optical parametric amplifiers for a broadband and flat gain with a low pump-tosignal noise transfer," J. Nonlinear Opt. Phys. \& Mater. 24, 1550038 (2015).

31. H. Pakarzadeh, R. Golabi, and C. Peucheret, "Two-pump fiber optical parametric amplifiers: Beyond the 6-wave model," Opt. Fiber Technol. 45, 223-230 (2018).

32. N. A. Silva, N. J. Muga, and A. N. Pinto, "Evolution of first-order sidebands from multiple fwm processes in hibi optical fibers," Opt. Commun. 284, 3408-3415 (2011).

33. C. J. McKinstrie, S. Radic, and M. Raymer, "Quantum noise properties of parametric amplifiers driven by two pump waves," Opt. Express 12, 5037-5066 (2004).

34. A. A. Albuquerque, B. J. Puttnam, J. M. Mendinueta, M. V. Drummond, S. Shinada, R. N. Nogueira, and N. Wada, "Experimental investigation of phase-sensitive amplification of data signals in a four-mode fiberbased psa," Opt. Lett. 40, 288-291 (2015).

35. W. Xie, I. Fsaifes, T. Labidi, and F. Bretenaker, "Investigation of degenerate dual-pump phase sensitive amplifier using multi-wave model," Opt. Express 23, 31896-31907 (2015).

36. J. Qian, M. Gao, L. Xiang, and G. Shen, "Investigation of high gain dual-pump phase sensitive amplifiers," Optik 135, 210-218 (2017).

37. M. Baillot, T. Chartier, and M. Joindot, "Multiple four-wave mixing in optical fibres," in 2014 The European Conference on Optical Communication (ECOC), (IEEE, 2014), pp. 1-3.
38. K. Inoue, "Influence of multiple four-wave-mixing processes on quantum noise of dual-pump phase-sensitive amplification in a fiber," J. Opt. Soc. Am. B 36, 1436-1446 (2019).

39. Y. Bouasria, D. Chatterjee, W. Xie, I. Fsaifes, F. Goldfarb, Y. Hassouni, and $\mathrm{F}$. Bretenaker, "Investigation of the noise figure in a degenerate dual-pump phase-sensitive amplifier using a multi-wave model," J. Opt. Soc. Am. B 37, 2745-2754 (2020).

40. M. Berry, "Pumping a swing revisited: minimal model for parametric resonance via matrix products," Eur. J. Phys. 39, 055007 (2018).

41. C. Chicone, Ordinary differential equations with applications (Springer Science \& Business Media, 2006).

42. S. Blanes, F. Casas, J.-A. Oteo, and J. Ros, "The magnus expansion and some of its applications," Phys. Reports 470, 151-238 (2009).

43. C. J. Joachain, Quantum collision theory (North-Holland, 1975).

44. C. Kittel and P. McEuen, Introduction to solid state physics, vol. 8 (Wiley New York, 1976)

45. M. Baillot, M. Gay, C. Peucheret, J. Michel, and T. Chartier, "Phase quadrature discrimination based on three-pump four-wave mixing in nonlinear optical fibers," Opt. Express 24, 26930-26941 (2016).

46. Z. Tong, C. Lundström, M. Karlsson, M. Vasilyev, and P. Andrekson, "Noise performance of a frequency nondegenerate phase-sensitive amplifier with unequalized inputs," Opt. Lett. 36, 722-724 (2011).

47. G. Van Simaeys, P. Emplit, and M. Haelterman, "Experimental study of the reversible behavior of modulational instability in optical fibers," $J$. Opt. Soc. Am. B 19, 477-486 (2002).

48. X.-M. Liu, "Theory and experiments for multiple four-wave-mixing processes with multifrequency pumps in optical fibers," Phys. Rev. A 77, 043818 (2008)

49. M. Marhic, N. Kagi, T.-K. Chiang, and L. Kazovsky, "Broadband fiber optical parametric amplifiers," Opt. Lett. 21, 573-575 (1996).

50. M. Gao, T. Kurosu, T. Inoue, and S. Namiki, "Efficient phase regeneration of dpsk signal by sideband-assisted dual-pump phase-sensitive amplifier," Electron. Lett. 49, 140-141 (2013).

51. W. Xie, I. Fsaifes, and F. Bretenaker, "Optimization of a degenerate dual-pump phase-sensitive optical parametric amplifier for all-optical regenerative functionality," Opt. Express 25, 12552-12565 (2017). 